\section{Condições de financiamento em saúde nos grandes municípios do Brasil}

\author{
Health financing conditions in large cities \\ in Brazil
}

Luciana Dias de Lima 1

Carla Lourenço Tavares de Andrade 1
${ }_{1}^{1}$ Escola Nacional de Saúde Pública Sergio Arouca, Fundação Oswaldo Cruz, Rio de Janeiro, Brasil.

Correspondência L. D. Lima

Departamento de Administração e Planejamento em Saúde, Escola Nacional de Saúde Pública Sergio Arouca, Fundação Oswaldo Cruz. Rua Leopoldo Bulhões 1480 Rio de Janeiro, $R J$ 21041-210, Brasil. luciana@ensp.fiocruz.br

\begin{abstract}
We evaluated the funding of the Brazilian Unified National Health System (SUS) in municipalities with more than 100,000 inhabitants. The main goal was to evaluate the impact of policies for health resource allocation within the municipal budget. A database was organized with information from revenues reported by municipalities in the Information System on Government Health Budgets (SIOPS) for the year 2005. Reported budgets were compared and correlated to the municipalities' geographic location. We conducted a cluster analysis to create more homogeneous groups according to health-related budget. The study showed a major variability among different regions and States, with varying degrees of municipal dependence on external funds. Although the large variability in sources may indicate multiple strategies for ensuring the necessary budget funds, the study suggests some barriers to public health funding in larger municipalities.
\end{abstract}

Health Financing; Health Policy, Single Health Sysytem

\section{Introdução}

Desde o início da década de 2000, os municípios brasileiros com mais de 100 mil habitantes têm sido priorizados pelo Ministério da Saúde em sua estratégia de expansão e consolidação da atenção básica, devido à grande concentração populacional em grandes centros urbanos e às baixas coberturas apresentadas pelo Programa Saúde da Família (PSF). Em que pese os vários problemas identificados a partir de pesquisas realizadas sobre o PSF em municípios de grande porte populacional e perfil metropolitano $1,2,3,4,5,6,7,8$, pouco ainda se sabe a respeito das condições financeiras que esses municípios apresentam para assumir sua responsabilidade plena na atenção básica.

Considera-se que decisões críticas dos governos municipais em relação às formas de organização e prestação do cuidado à saúde são condicionadas pela disponibilidade de recursos e pela importância relativa das várias fontes de receitas vinculadas à saúde em seus orçamentos 9,10. Isso porque, devido à importância relativa dos diferentes tipos de recursos vinculados, variações na composição orçamentária refletem: diversidade na disponibilidade de receitas voltadas para saúde; oportunidades diferenciadas de obtenção e expansão de receitas e relações intergovernamentais predominantes; tipos de despesa que podem ser efetivadas e maior ou menor autonomia na alocação dos recursos. 
Nesse sentido, duas ordens de fatores importam para compreensão das condições de financiamento do Sistema Único de Saúde (SUS) nos municípios de grande porte populacional.

A primeira está relacionada à forma como se estruturam a divisão das competências tributárias e os dispositivos constitucionais que determinam a partilha fiscal obrigatória entre as distintas esferas de governo (particularmente, da União e dos estados para os municípios). Esses recursos constituem as receitas próprias dos governos municipais que se vinculam à saúde por meio das diretrizes da Emenda Constitucional $n^{o} .29$ (EC-29) de 2000.

A segunda refere-se às regras intrínsecas à política nacional de saúde, regulamentadas ou não por meio de instrumentos legais e normativos, que atuam sobre os recursos setoriais. Essas receitas diferenciam-se por terem um destino explícito, sendo orientadas exclusivamente para a saúde. Elas incluem os diferentes tipos de transferências intergovernamentais oriundas dos Fundos de Saúde (automáticas ou realizadas mediante a celebração de convênios) e as receitas decorrentes da prestação direta de serviços.

Tem-se como hipótese que os municípios brasileiros com mais de 100 mil habitantes apresentam perfis variados de receitas públicas que devem ser destinadas ao financiamento do SUS, como resultado do processo de descentralização tributária previsto na Constituição de 1988 e da redistribuição fiscal proporcionada, principalmente, pelas transferências federais do SUS. As características das receitas vinculadas à saúde diferenciam os municípios segundo graus de dependência - fiscal e setorial -, em relação à União e aos estados, para obtenção e expansão de recursos financeiros, com possíveis implicações para o desempenho da gestão local, as relações intergovernamentais, a integração e a prestação de serviços.

Outra hipótese investigada é a de que as regras que determinam a vinculação de recursos para a saúde no Brasil, em seu conjunto, geram constrangimentos e desequilíbrios federativos e não favorecem a diminuição das desigualdades nas condições de financiamento em saúde dos municípios mais populosos.

A avaliação do financiamento do SUS sob a ótica dos governos municipais é um campo a ser explorado nas pesquisas sobre o tema. Em países federativos, quando se observam fluxos de transferências intergovernamentais a partir do orçamento cedente, nem sempre se consegue dimensionar o impacto mais amplo que eles têm sobre o universo dos orçamentos locais que se beneficiam da obtenção de recursos.
Assim, justifica-se o estudo empírico realizado no âmbito dessa pesquisa. Partindo da concepção de atenção básica como estratégia de reordenamento e articulação do conjunto de ações e serviços que compõem o sistema de saúde 11,12, procurou-se investigar as condições de financiamento do SUS nos municípios com mais de 100 mil habitantes de forma ampla. A metodologia adotada destaca os resultados das regras (critérios e condicionantes) que interferem na distribuição, apropriação e uso de recursos vinculados à saúde nos orçamentos municipais.

\section{Material e métodos}

Para o alcance do objetivo pretendido, foi constituída uma base de dados contendo, como variáveis, os valores monetários (em moeda corrente nacional) de todos os ingressos auferidos durante o ano de 2005 pelos municípios com mais de 100 mil habitantes. Os dados foram coletados a partir do Sistema de Informações sobre Orçamentos Públicos em Saúde (SIOPS).

A escolha do ano de 2005 permitiu averiguar os efeitos da EC-29 e do modelo de financiamento descentralizado da saúde consolidado a partir da segunda metade da década de 1990, que introduziu novas formas de repasse de recursos federais para as esferas locais no que se refere à atenção básica e demais níveis de complexidade do sistema (Piso de Atenção Básica Fixo - PAB Fixo; Piso de Atenção Básica Variável - PAB Variável; Fundo de Ações Estratégicas e de Compensação - FAEC).

Os efeitos do federalismo fiscal e das transferências de recursos do SUS são analisados comparando-se e correlacionando-se os valores das receitas públicas observadas nos orçamentos municipais 13. Para permitirem-se comparações entre os municípios, as receitas informadas foram transformadas em valores per capita. Em seguida, procedeu-se à identificação das receitas vinculadas à saúde pelas regras vigentes em 2005. As receitas correntes voltadas para o financiamento da saúde foram classificadas em duas categorias:

(i) Receitas com destino vinculado à saúde (receitas setoriais ou exclusivas da saúde): recursos cuja classificação do destino é explicitada no ingresso orçamentário e que cumprem funções de financiamento exclusivo das ações e serviços descentralizados do SUS;

(ii) Receitas vinculadas à saúde por determinação da EC-29 de 2000 e regulamentação complementar: recursos próprios (diretamente arrecadados ou adquiridos por meio das transferências constitucionais da União e dos estados) que, por 
determinação legal, constituem base vinculável à saúde no momento da execução das despesas orçamentárias. Aplicou-se sobre essas receitas o percentual mínimo de vinculação preconizado pela EC-29 para os municípios (15\%).

Para além da análise descritiva dos dados no conjunto de municípios e nos agrupamentos formados segundo localização geográfica (região e estados), verificou-se a distribuição proporcional dos diferentes componentes das receitas municipais per capita e a importância relativa das transferências automáticas do SUS provenientes do Fundo Nacional de Saúde. Adotou-se a razão de medianas para medir as distâncias entre os valores de determinadas fontes de receitas observadas nos grupos de municípios e o agregado nacional. A mediana foi a medida de tendência central adotada dado a elevada dispersão dos valores observados.

O impacto da vinculação orçamentária para cada município foi calculado considerando a proporção das receitas, nas duas categorias adotadas - receitas com destino vinculado à saúde e receitas vinculadas à saúde segundo o estabelecido pela EC-29 - na receita corrente total. A disponibilidade total dos recursos destinados à saúde assim como a capacidade de redistribuição e suplementação fiscal proporcionada pelas diferentes fontes de receitas vinculadas à saúde também foram mensuradas.

Para a conformação de grupos homogêneos de municípios de grande porte, segundo distintas condições de financiamento em saúde, utilizouse a técnica de análise de agrupamentos (cluster analysis). Essa técnica é uma ferramenta de análise exploratória de dados que consiste em ordenar diferentes objetos em grupos de modo que o grau de associação entre dois objetos é máximo se eles pertencem ao mesmo grupo e mínimo caso contrário. Os agrupamentos são realizados baseados nos conceitos de similaridade ou de distância (dissimilaridade) 14 .

Os aglomerados são formados através da menor distância Euclidiana entre os casos, e o número de aglomerados é definido pelo critério BIC (Schwarz Bayesian Criterion), ou seja, quando a mudança no BIC é pequena, o procedimento seleciona tantos aglomerados quantos forem criados até o momento.

Os indicadores utilizados para a construção dos agrupamentos exprimem graus de dependência variados dos municípios em relação às principais fontes de receitas correntes municipais vinculadas ao financiamento do SUS. Foram construídos três indicadores para a diferenciação dos municípios:

(i) Grau de dependência orçamentária dos recursos da EC-29: expressa o quanto o município de- pende financeiramente de seus recursos próprios para o financiamento do SUS. O indicador foi calculado considerando a proporção de todas as receitas que compõem a base vinculável da saúde definida pela EC-29 no total das receitas vinculadas à saúde;

(ii) Grau de dependência orçamentária dos recursos do $P A B$ : esse indicador mede a dependência financeira do município em relação aos recursos voltados exclusivamente para o financiamento da atenção básica no SUS. No cálculo do indicador, considerou-se a participação percentual do somatório dos ingressos orçamentários municipais relacionados às transferências federais voltadas para a atenção básica (PAB Fixo e Variável) no total das receitas vinculadas à saúde;

(iii) Grau de dependência orçamentária dos recursos voltados para a média e alta complexidade: indica o quanto o município é dependente financeiramente dos recursos voltados para o financiamento das ações e serviços de média e alta complexidade do SUS, adquiridos por meio da contraprestação de serviços de saúde ou, no caso dos municípios habilitados na condição de gestão plena do sistema municipal, transferidos diretamente pelo Ministério da Saúde. De forma semelhante, calculou-se a proporção do conjunto dessas receitas no total das receitas vinculadas à saúde.

O programa utilizado para a organização, processamento e análise estatística dos dados foi o pacote estatístico SPSS, versão 13.0 (SPSS Inc., Chicago, Estados Unidos).

\section{Resultados e discussão}

A participação relativa de cada componente da receita corrente varia nos grupos de municípios segundo localização geográfica (Figura 1). Em média, as principais fontes de receitas correntes municipais nas distintas regiões do país são as transferências intergovernamentais provenientes dos estados e da União, com destaque para o Imposto sobre Circulação de Mercadorias e Serviços (ICMS) e o Fundo de Participação dos Municípios (FPM). No entanto, enquanto as transferências dos estados e a receita tributária própria (tributos diretamente arrecadados) são mais relevantes nos orçamentos dos municípios situados nas regiões Sudeste e Sul, no Norte, Nordeste e Centro-Oeste, a importância das transferências da União é mais significativa. Os municípios das regiões Nordeste e Norte também se diferenciam pela maior proporção de receitas obtidas das transferências vinculadas à educação por meio do Fundo Nacional de Desenvolvimento do Ensino Fundamental e Valorização do Magistério (FUNDEF). 


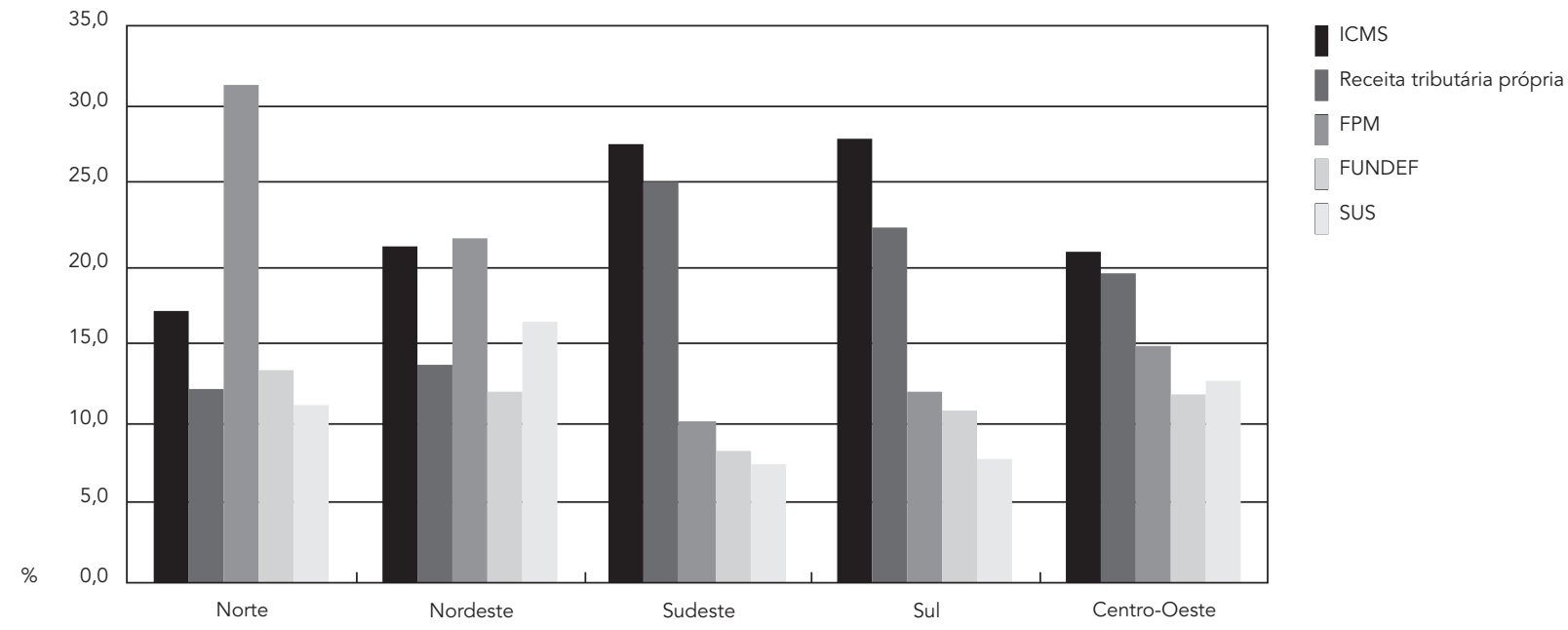

SUS: Sistema Único de Saúde; FUNDEF: Fundo Nacional de Desenvolvimento do Ensino Fundamental e Valorização do Magistério; ICMS: Imposto sobre Circulação de Mercadorias e Serviços; FPM: Fundo de Participação dos Municípios.

Fonte: Sistema de Informações sobre Orçamentos Públicos em Saúde, Departamento de Informática do SUS, Ministério da Saúde.

As diferenças na composição orçamentária dos municípios por estados também são significativas. Em relação à arrecadação tributária e as transferências estaduais, destacam-se os municípios de São Paulo e dos estados do Sul do país. No que se refere às transferências da União, sobressaem-se os municípios de Boa Vista (Roraima) e os localizados em Alagoas, Maranhão, Tocantins e Pará.

Em geral, observa-se o seguinte comportamento dos municípios em relação às principais fontes de receitas correntes em 2005: (1) as receitas provenientes da cota-parte do ICMS e da receita tributária própria são mais expressivas nos municípios situados nas regiões Sudeste, Sul, seguidos do Centro-Oeste; (2) nas regiões CentroOeste e Nordeste, os valores das transferências federais do SUS são maiores do que nas demais regiões do país; e (3) municípios das regiões Norte e Nordeste se destacam em relação aos recursos mais elevados provenientes do FPM.

Na Tabela 1, pode-se perceber o quão relevantes são as transferências federais do SUS para os municípios com mais de 100 mil habitantes. Considerando-se o universo dos municípios do Nordeste, verifica-se que, no mínimo, $68 \%$ dos municípios ali situados possuem receita oriunda das transferências SUS superiores à arrecadação tributária própria. Em alguns estados, essa proporção chega a 100\%, como na Paraíba e no Piauí. Na Região Norte, no mínimo, em $47 \%$ dos municípios existentes em 2005, o SUS é mais importante do que a arrecadação. O Sudeste e o Sul são as regiões onde as transferências SUS são menos significativas em relação à arrecadação. No Centro-Oeste, chama a atenção o Estado de Goiás, onde as transferências SUS são mais importantes do que a receita de tributos diretamente arrecadados em 50\% dos municípios ali situados.

Semelhantemente, considerando-se as transferências do FUNDEF, da Cota-parte do ICMS e da Cota-parte do FPM, é possível averiguar que: (1) no Nordeste e no Centro-Oeste, o número de municípios que têm ingresso orçamentário proveniente do SUS maior do que o FUNDEF é mais significativo que as demais regiões; (2) nas regiões Nordeste e Norte, chamam atenção as proporções de municípios que têm receita SUS superior ao ICMS; e (3) as regiões Centro-Oeste, Sudeste e Sul são aquelas que apresentam maior número de municípios com ingresso do SUS superior ao FPM.

A importância do SUS aos cofres públicos nos revela o quanto as economias dos grandes municípios do país são dependentes das transferências de recursos da saúde provenientes da União. 
Distribuição percentual dos municípios por importância do SUS como fonte de receita corrente para os municípios com mais de 100 mil habitantes. Brasil, 2005.

\begin{tabular}{|c|c|c|c|c|c|c|c|c|c|}
\hline \multirow[t]{2}{*}{ Região/UF } & \multicolumn{2}{|c|}{$\begin{array}{l}\text { Municípios com receita } \\
\text { de transferência federal } \\
\text { do SUS maior que a } \\
\text { arrecadação tributária } \\
\text { própria }\end{array}$} & \multicolumn{2}{|c|}{$\begin{array}{l}\text { Municípios com receita } \\
\text { de transferência federal } \\
\text { do SUS maior que } \\
\text { o FUNDEF }\end{array}$} & \multicolumn{2}{|c|}{$\begin{array}{c}\text { Municípios com receita } \\
\text { de transferência federal } \\
\text { do SUS maior que } \\
\text { o ICMS }\end{array}$} & \multicolumn{2}{|c|}{$\begin{array}{c}\text { Municípios com receita } \\
\text { de transferência federal } \\
\text { do SUS maior que } \\
\text { o FPM }\end{array}$} & \multirow{2}{*}{$\begin{array}{c}\text { Número de } \\
\text { municípios } \\
\text { n }\end{array}$} \\
\hline & $\mathrm{n}$ & $\%$ & $\mathrm{n}$ & $\%$ & $\mathrm{n}$ & $\%$ & $n$ & $\%$ & \\
\hline \multicolumn{10}{|l|}{ Norte } \\
\hline$A C$ & - & - & - & - & - & - & - & - & 1 \\
\hline$A P$ & - & - & - & - & - & - & - & - & 1 \\
\hline$A M$ & 1 & 50,0 & - & - & - & - & - & - & 2 \\
\hline PA & 6 & 75,0 & 3 & 37,5 & 6 & 75,0 & 2 & 25,0 & 8 \\
\hline $\mathrm{RO}$ & 1 & 50,0 & 1 & 50,0 & 1 & 50,0 & 1 & 50,0 & 2 \\
\hline $\mathrm{RR}$ & - & - & 1 & 100,0 & - & - & - & - & 1 \\
\hline TO & - & - & - & - & - & - & - & - & 2 \\
\hline Subtotal & 8 & 47,1 & 5 & 29,4 & 7 & 41,2 & 3 & 17,6 & 17 \\
\hline \multicolumn{10}{|l|}{ Nordeste } \\
\hline $\mathrm{AL}$ & 1 & 50,0 & 2 & 100,0 & 2 & 100,0 & - & - & 2 \\
\hline BA & 9 & 60,0 & 8 & 53,3 & 7 & 46,7 & 3 & 20,0 & 15 \\
\hline $\mathrm{CE}$ & 6 & 85,7 & 4 & 57,1 & 4 & 57,1 & 2 & 28,6 & 7 \\
\hline MA & 6 & 85,7 & 4 & 57,1 & 5 & 71,4 & 2 & 28,6 & 7 \\
\hline PB & 3 & 100,0 & 2 & 66,7 & 2 & 66,7 & 1 & 33,3 & 3 \\
\hline PE & 6 & 60,0 & 6 & 60,0 & 2 & 20,0 & - & - & 10 \\
\hline $\mathrm{Pl}$ & 2 & 100,0 & 1 & 50,0 & 1 & 50,0 & 1 & 50,0 & 2 \\
\hline $\mathrm{RN}$ & 1 & 33,3 & 2 & 66,7 & - & - & 1 & 33,3 & 3 \\
\hline SE & 1 & 50,0 & 2 & 100,0 & 1 & 50,0 & 1 & 50,0 & 2 \\
\hline Subtotal & 35 & 68,6 & 31 & 60,8 & 24 & 47,1 & 11 & 21,6 & 51 \\
\hline \multicolumn{10}{|l|}{ Sudeste } \\
\hline ES & 1 & 11,1 & - & - & - & - & 1 & 11,1 & 9 \\
\hline MG & 8 & 30,8 & 16 & 61,5 & 9 & 34,6 & 13 & 50,0 & 26 \\
\hline RJ & 5 & 22,7 & 4 & 18,2 & 4 & 18,2 & 11 & 50,0 & 22 \\
\hline $\mathrm{SP}$ & - & - & 31 & 43,7 & 2 & 2,8 & 21 & 29,6 & 71 \\
\hline Subtotal & 14 & 10,9 & 51 & 39,8 & 15 & 11,7 & 46 & 35,9 & 128 \\
\hline \multicolumn{10}{|l|}{ Sul } \\
\hline PR & 1 & 6,3 & 4 & 25,0 & 4 & 25,0 & 4 & 25,0 & 16 \\
\hline RS & 1 & 5,3 & 4 & 21,1 & 1 & 5,3 & 4 & 21,1 & 19 \\
\hline SC & 1 & 10,0 & 6 & 60,0 & - & - & 5 & 50,0 & 10 \\
\hline Subtotal & 3 & 6,7 & 14 & 31,1 & 5 & 11,1 & 13 & 28,9 & 45 \\
\hline \multicolumn{10}{|l|}{ Centro-Oeste } \\
\hline GO & 3 & 50,0 & 3 & 50,0 & 3 & 50,0 & 3 & 50,0 & 6 \\
\hline MT & 1 & 33,3 & 2 & 66,7 & 1 & 33,3 & 1 & 33,3 & 3 \\
\hline MS & - & - & 2 & 66,7 & - & - & 2 & 66,7 & 3 \\
\hline Subtotal & 4 & 33,3 & 7 & 58,3 & 4 & 33,3 & 6 & 50,0 & 12 \\
\hline Total & 64 & 25,3 & 108 & 42,7 & 55 & 21,7 & 79 & 31,2 & 253 \\
\hline
\end{tabular}

SUS: Sistema Único de Saúde; UF: Unidade da Federação; FUNDEF: Fundo Nacional de Desenvolvimento do Ensino Fundamental e Valorização do Magistério; ICMS: Imposto sobre Circulação de Mercadorias e Serviços; FPM: Fundo de Participação dos Municípios; AC: Acre; AL: Alagoas; AM: Amazonas; AP: Amapá; BA: Bahia; CE: Ceará; ES: Espírito Santo; GO: Goiás; MA: Maranhão; MG: Minas Gerais; MT: Mato Grosso; MS: Mato Grosso do Sul; PA: Pará; PB: Paraíba; PE: Pernambuco; PI: Piauí; PR: Paraná; RJ: Rio de Janeiro; RN: Rio Grande do Norte; RO: Rondônia; RR: Roraima; RS: Rio Grande do Sul; SE: Sergipe; SC: Santa Catarina; SP: São Paulo; TO: Tocantins.

Fonte: Sistema de Informações sobre Orçamentos Públicos em Saúde, Departamento de Informática do SUS, Ministério da Saúde. 
A relação entre os recursos legalmente transferidos nos diversos agrupamentos de municípios por localização geográfica e a mediana nacional é expressa na Tabela 2. Tal como sugerido por Prado 15, a capacidade de redistribuição fiscal de uma dada transferência intergovernamental está associada aos critérios de rateio utilizados que as associam mais ou menos à distribuição irregular das bases tributárias locais. Esta pode ser avaliada comparando-se a disponibilidade dos recursos transferidos com aqueles oriundos da arrecadação tributária própria.

O ICMS, que na sua maior parte (75\%) é um fluxo devolutivo, possui forte associação com a base produtiva local e segue o padrão da arrecadação direta de tributos. Com capacidade redistributiva fiscal mais limitada, esse tributo é fundamental nas regiões e estados mais desenvolvidos do ponto de vista econômico, criando uma relação maior de dependência e aproximando os governos locais das instâncias estaduais.

No caso do FPM, os critérios de partilha privilegiam o Nordeste e o Norte. Há de se destacar, contudo, que as diferenças na apropriação da cota-parte do FPM são menos expressivas entre as regiões do que aquelas decorrentes unicamente da disponibilidade das bases tributárias.

Em relação às transferências federais do SUS, o que tudo indica é que a ampla variedade de critérios consolidados na segunda metade da década de 1990 (população, cobertura de programas, oferta e produção de serviços, entre outros) tende a redistribuir recursos entre municípios de grande porte populacional situados em territórios com padrões de desenvolvimento diferenciados, privilegiando, de forma mais significativa, as regiões Centro-Oeste e Nordeste do país.
O padrão regional de distribuição das receitas correntes municipais se reflete nas taxas de vinculação orçamentária à saúde. Os municípios situados no Nordeste são os que apresentam, em média, maior proporção de receitas vinculadas à saúde (cerca de $26 \%$ do orçamento corrente), com destaque para os localizados na Paraíba e em Alagoas, que apresentam taxas de vinculação superiores a $30 \%$.

Observa-se que as diferenças entre os municípios agregados por região e estado são mais significativas quando as receitas vinculadas à saúde são desdobradas. A maior importância dos recursos exclusivos da saúde encontra-se nos municípios situados no Nordeste, Centro-Oeste e Norte. No Nordeste, eles representam $66,7 \%$ da receita corrente total vinculada à saúde; no Centro-Oeste, $63 \%$; e no Norte, $56 \%$. É, portanto, o maior aporte de recursos setorialmente orientados que explica o grau relativamente mais elevado de vinculação à saúde nessas regiões. $\mathrm{O}$ efeito da EC-29 na apropriação de recursos para a saúde é mais significativo nos municípios situados nas regiões centro-sul do país, tendo em vista a maior importância dos recursos próprios nas receitas correntes totais desses municípios.

O estudo evidencia que, em muitos casos, um grau elevado de vinculação à saúde das receitas correntes municipais pode ser necessário para gerar condições de financiamento semelhantes nos municípios com mais de 100 mil habitantes. Os municípios localizados nas regiões Sudeste e Centro-Oeste são os que apresentam os maiores valores, médios e medianos, de receitas totais per capita vinculadas à saúde, seguidos daqueles situados nas regiões Nordeste e Sul. A situação mais crítica parece ser a dos municípios situados no Norte do país, pois o ingresso de recursos

Tabela 2

Razão entre os valores medianos observados das receitas municipais per capita relativas - arrecadação tributária própria, Fundo de Participação dos Municípios (FPM), Imposto sobre Circulação de Mercadorias e Serviços (ICMS), Sistema Único de Saúde (SUS) e Fundo Nacional de Desenvolvimento do Ensino Fundamental e Valorização do Magistério (FUNDEF). Brasil, 2005.

\begin{tabular}{lccccc}
\hline Região & $\begin{array}{c}\text { Receita } \\
\text { tributária } \\
\text { própria }\end{array}$ & ICMS & FPM & FUNDEF & SUS \\
\hline Norte & 45,6 & 55,7 & 105,4 & 103,9 & 93,4 \\
Nordeste & 38,0 & 58,4 & 119,6 & 97,8 & 134,9 \\
Sudeste & 121,8 & 118,5 & 97,5 & 91,3 & 90,1 \\
Sul & 115,1 & 108,1 & 93,8 & 111,1 & 42,9 \\
Centro-Oeste & 92,3 & 98,1 & 99,7 & 103,8 & 141,1 \\
Total & 100,0 & 100,0 & 100,0 & 100,0 & 100,0 \\
\hline
\end{tabular}

Fonte: Sistema de Informações sobre Orçamentos Públicos em Saúde, Departamento de Informática do SUS, Ministério da Saúde (receitas orçamentárias) e Instituto Brasileiro de Geografia e Estatística (população estimada). 
exclusivos da saúde não é suficiente para compensar os baixos valores das receitas vinculadas à saúde pela EC-29.

Detalhando-se um pouco mais as fontes de recursos por região, observa-se que o principal componente das transferências federais do SUS está voltado para a média e alta complexidade nas diversas regiões do país. No entanto, no Nordeste e, principalmente, na Região Norte, destacam-se os recursos voltados para a atenção básica (respectivamente, $32 \%$ e $46 \%$ das transferências federais do SUS). Isso é explicado não somente pelo processo de implantação e expansão do PSF, mas também pela menor capacidade de oferta de serviços do SUS de maior complexidade nessas regiões.

Frente às diferenças orçamentárias encontradas, a aplicação da técnica de análise de agrupamentos (cluster analysis) propiciou a geração de quatro aglomerados de municípios com condições de financiamento do SUS diversas:

- Aglomerado 1: alto grau de dependência orçamentária dos recursos próprios vinculados à saúde pela EC-29 com baixa participação daqueles voltados para a média e alta complexidade. Esse grupo é composto por 73 municípios que se destacam dos demais pela maior participação dos recursos próprios no conjunto da receita vinculada à saúde em seus orçamentos. Esses municípios também apresentam o menor grau de dependência em relação aos recursos voltados para a média e alta complexidade repassados por outros níveis de governo. A proporção de receitas voltadas para o financiamento exclusivo da atenção básica não foi determinante para a configuração desse agrupamento.

- Aglomerado 2: baixo grau de dependência orçamentária dos recursos voltados para atenção básica no SUS. Engloba 74 municípios que se destacam pelo menor grau de dependência em relação aos recursos do $\mathrm{PAB}$ transferidos pelo Ministério da Saúde e por médias relativamente altas em relação à sua dependência aos recursos próprios e àqueles voltados para a média e alta complexidade no SUS.

- Aglomerado 3: alto grau de dependência orçamentária dos recursos voltados para atenção básica no SUS. Agrega 29 municípios que se diferenciam pelo maior grau de dependência em relação aos recursos do $\mathrm{PAB}$ e por médias relativamente baixas referentes à participação dos recursos próprios e daqueles voltados para a média e alta complexidade no total de recursos vinculados à saúde.

- Aglomerado 4: baixo grau de dependência orçamentária dos recursos próprios vinculados à saúde pela EC-29 com alta participação daqueles voltados para a média e alta complexidade. Con- tém 77 municípios que se destacam pela menor participação dos recursos próprios no total da receita vinculada à saúde e pela maior média no que se refere à proporção dos recursos voltados para a média e alta complexidade, apresentando, ainda, baixo grau de dependência em relação aos recursos transferidos pelo Ministério da Saúde para a atenção básica.

A Figura 2 expressa a distribuição espacial dos municípios segundo os aglomerados, e a Tabela 3 resume as principais características desses agrupamentos.

\section{Conclusões}

O trabalho procurou responder as seguintes questões: como os municípios com mais de 100 mil habitantes se diferenciam em relação às suas condições de financiamento em saúde? Quais os efeitos das regras que interferem na distribuição de recursos vinculados (fiscais e setoriais) para o financiamento do SUS nos municípios com mais de 100 mil habitantes?

Em primeiro lugar, os achados confirmam a hipótese de que relações fiscais e orçamentárias que se estabelecem entre os governos na federação brasileira são ineficazes do ponto de vista da redução das desigualdades que se verificam entre municípios com grande porte populacional situados em distintas regiões e estados do país. As receitas municipais vinculadas à saúde das regiões Norte e Nordeste permanecem bem aquém das verificadas nas regiões mais ao sul do país. Além disso, a heterogeneidade das finanças públicas municipais no interior dos estados é também expressiva. A manutenção da iniqüidade geográfica em relação à renda e à apropriação econômica resulta em condições bastante desfavoráveis do ponto de vista da capacidade local de financiamento das ações e serviços de saúde e indicam outras formas de agregação para diferenciação dos municípios.

No entanto, os dados sugerem uma situação de destaque dos municípios da Região CentroOeste que apresentam características orçamentárias que os aproximam daqueles localizados no eixo mais dinâmico e desenvolvido do país, o que pode ser visto quando as receitas públicas municipais são analisadas com mais detalhe.

Em segundo lugar, ressalta-se a importância das transferências federais do SUS como fonte regular de recursos orçamentários para os municípios brasileiros com mais de $100 \mathrm{mil}$ habitantes. No ano de 2005, as transferências federais do SUS representam, nos orçamentos dos executivos locais a: (1) quinta principal fonte de receita tributária, abaixo da Cota-parte do ICMS, 


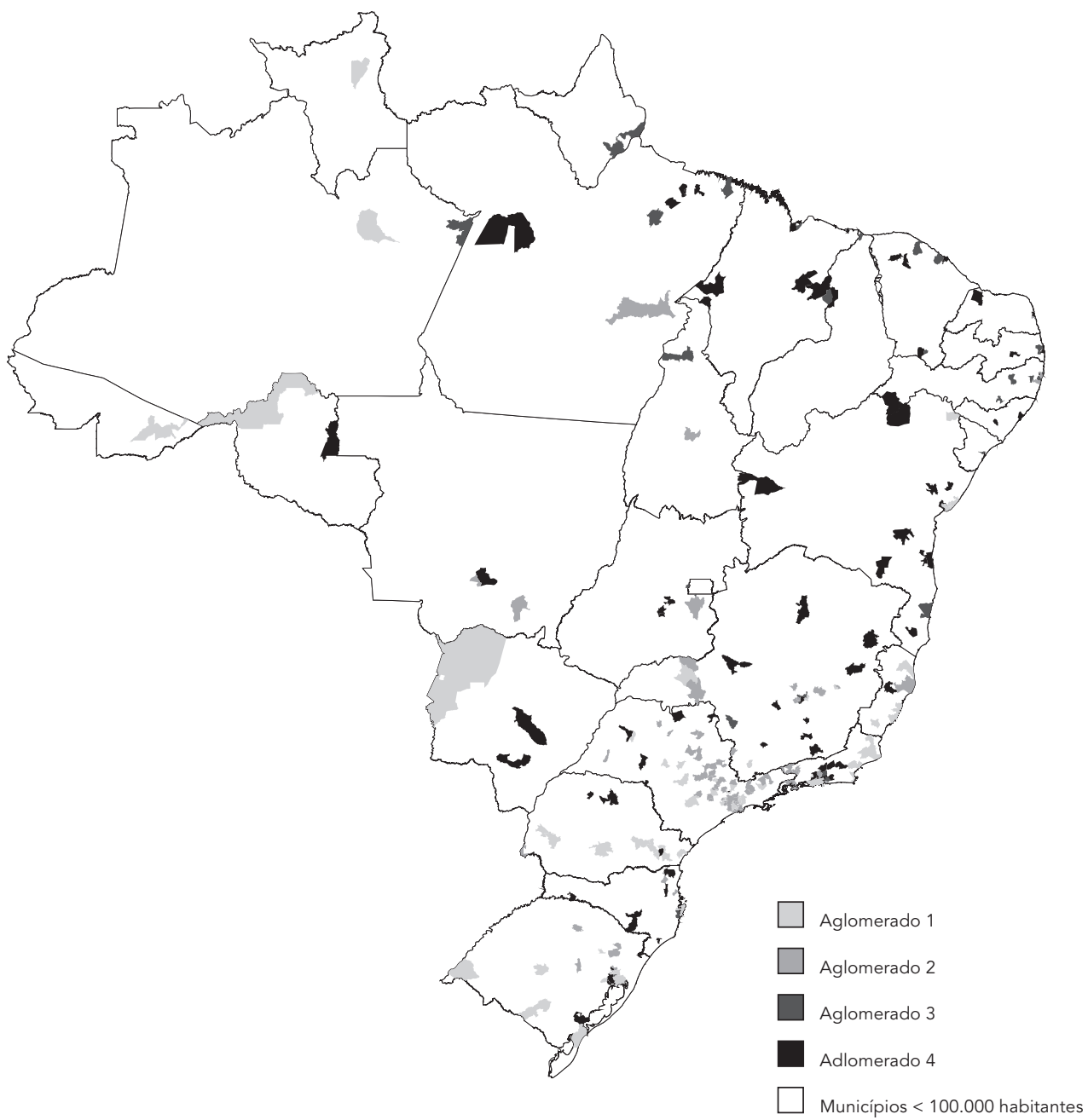

da arrecadação tributária própria, do FPM, e do Fundo Nacional de Desenvolvimento do Ensino Fundamental e Valorização do Magistério (FUNDEF); (2) segunda principal transferência regular da União (abaixo do FPM); (3) fonte de receita mais importante do que a arrecadação tributária própria em, pelo menos, $25 \%$ dos municípios.

As transferências federais do SUS, se comparadas com a arrecadação, são particularmente significativas nos municípios situados nas regiões Nordeste e Norte do país. A importância desses recursos reforça o papel indutor e coordenador do Ministério da Saúde, representando um forte estímulo à adesão municipal a políticas federais atreladas a recursos financeiros nessas localidades.

De fato, desde a segunda metade da década de 1990, o Ministério da Saúde tem lançado mão de diversos instrumentos para modelar a assistência à saúde prestada nos municípios brasileiros 9. Por meio do PAB Variável, por exemplo, vários programas federais passam a ser fontes adicionais de receitas municipais. Mudanças nos critérios de distribuição desses recursos também podem influenciar a decisão quanto ao momento e magnitude da implantação e expansão do programa pelo gestor local, como, por exemplo, o PSF. 
Principais características dos aglomerados municipais. Brasil, 2005

\begin{tabular}{|c|c|c|c|c|c|}
\hline Características & Aglomerado 1 & Aglomerado 2 & Aglomerado 3 & Aglomerado 4 & Total \\
\hline $\begin{array}{l}\text { Municípios com mais } \\
\text { de } 100.000 \text { habitantes }\end{array}$ & $73(28,9 \%)$ & $74(29,2 \%)$ & $29(11,5 \%)$ & $77(30,4 \%)$ & $253(100,0 \%)$ \\
\hline População & $25.400 .315(26,5 \%)$ & $32.456 .252(33,8 \%)$ & $6.365 .372(6,6 \%)$ & $31.708 .188(33,1 \%)$ & $95.930 .127(100,0 \%)$ \\
\hline $\begin{array}{l}\text { Faixas populacionais } \\
\text { em que o grupo } \\
\text { é preponderante }\end{array}$ & $\begin{array}{c}<200.000(33,3 \%) \\
200.001-400.000 \\
(31,2 \%)\end{array}$ & - & - & $\begin{array}{c}400.001-1.000 .000 \\
(50,0 \%) ;>1.000 .000 \\
(46,2 \%)\end{array}$ & - \\
\hline $\begin{array}{l}\text { Principais regiões onde } \\
\text { o grupo se localiza }\end{array}$ & SE $(54,8 \%) ; S(31,5 \%)$ & SE $(78,4 \%)$ & $\begin{array}{c}\text { NE }(48,3 \%) ; \text { SE } \\
(20,7 \%) ; N(17,2 \%)\end{array}$ & $\begin{array}{c}\text { NE }(37,7 \%) ; \mathrm{SE} \\
(31,2 \%)\end{array}$ & - \\
\hline $\begin{array}{l}\text { Regiões onde o grupo } \\
\text { é preponderante }\end{array}$ & $\mathrm{S}(51,1 \%)$ & SE $(45,3 \%)$ & - & $\begin{array}{l}\text { NE }(56,9 \%) ; C O \\
(50 \%) ; N(35,3 \%)\end{array}$ & - \\
\hline $\begin{array}{l}\text { Principais estados onde } \\
\text { o grupo se localiza }\end{array}$ & $\begin{array}{c}\mathrm{SP}(31,5 \%) ; \mathrm{PR} \\
(15,1 \%) ; \mathrm{RS} 13,7 \%)\end{array}$ & $\begin{array}{c}\mathrm{SP}(56,8 \%) ; \mathrm{MG} \\
(10,8 \%)\end{array}$ & $\begin{array}{c}\text { PE (17,2\%); MG } \\
(13,8 \%) ; C E(10,3 \%)\end{array}$ & $\begin{array}{c}\text { MG }(13 \%) ; B A \\
(11,7 \%)\end{array}$ & - \\
\hline Municípios capitais & $8(30,8 \%)$ & $3(11,5 \%)$ & $1(3,9 \%)$ & $14(53,8 \%)$ & $26(100,0 \%)$ \\
\hline Capitais & $\begin{array}{l}\text { Porto Velho; Rio } \\
\text { Branco; Manaus; } \\
\text { Boa Vista; Salvador; } \\
\text { Vitória; Rio de } \\
\text { Janeiro; Florianópolis }\end{array}$ & $\begin{array}{c}\text { Palmas; Recife; São } \\
\text { Paulo }\end{array}$ & Macapá & $\begin{array}{c}\text { Belém; São Luís; } \\
\text { Teresina; Fortaleza; } \\
\text { Natal; João Pessoa; } \\
\text { Maceió; Aracaju; } \\
\text { Belo Horizonte; } \\
\text { Curitiba; Porto } \\
\text { Alegre; Campo } \\
\text { Grande; Cuiabá; } \\
\text { Goiânia }\end{array}$ & - \\
\hline $\begin{array}{l}\text { Municípios integrantes de } \\
\text { Região Metropolitana }\end{array}$ & $41(33,9 \%)$ & $35(28,9 \%)$ & $15(12,4 \%)$ & $30(24,8 \%)$ & $121(100,0 \%)$ \\
\hline $\begin{array}{l}\text { Municípios habilitados em } \\
\text { gestão plena do sistema } \\
\text { municipal }\end{array}$ & $13(7,6 \%)$ & $67(39,0 \%)$ & $16(9,2 \%)$ & $76(44,2 \%)$ & $172(100,0 \%)$ \\
\hline $\begin{array}{l}\text { Condição de habilitação que } \\
\text { predomina no grupo }\end{array}$ & $\begin{array}{l}\text { Não habilitado em } \\
\text { GPSM }(82,2 \%)\end{array}$ & $\begin{array}{l}\text { Habilitado em } \\
\text { GPSM }(90,5 \%)\end{array}$ & $\begin{array}{l}\text { Habilitado em } \\
\text { GPSM }(55,2 \%)\end{array}$ & $\begin{array}{l}\text { Habilitado em } \\
\text { GPSM }(98,7 \%)\end{array}$ & - \\
\hline
\end{tabular}

GPSM: Gestão Plena do Sistema Municipal; BA: Bahia; CO: Centro-Oeste; MG: Minas Gerais; N: Norte; NE: Nordeste; PE: Pernanbuco; PR: Paraná; RS: Rio Grande do Sul; S: Sul; SE: Sudeste; SP: São Paulo.

Notas: Aglomerado 1: alto grau de dependência orçamentária dos recursos próprios vinculados à saúde pela EC-29 com baixa participação daqueles voltados para a média e alta complexidade; Aglomerado 2: baixo grau de dependência orçamentária dos recursos voltados para atenção básica no SUS; Aglomerado 3: alto grau de dependência orçamentária dos recursos voltados para atenção básica no SUS; Aglomerado 4: baixo grau de dependência orçamentária dos recursos próprios vinculados à saúde pela EC-29 com alta participação daqueles voltados para a média e alta complexidade.

Fonte: Sistema de Informações sobre Orçamentos Públicos em Saúde, Departamento de Informática do SUS, Ministério da Saúde.

Por sua vez, alterações nos valores financeiros das Tabelas de Procedimentos do SUS, utilizadas como referência para pagamentos dos prestadores, repercutem nos tetos financeiros dos municípios e nos montantes transferidos para a média e alta complexidade, o que tende a modificar o padrão de oferta e cobertura de serviços locais.

Depreende-se que o poder indutor do Ministério da Saúde pode ser favorecido pela maior situação de dependência financeira dos municípios às diferentes modalidades de transferências intergovernamentais na saúde.
A pesquisa diferenciou os municípios com mais de 100 mil habitantes segundo sua situação de dependência orçamentária às principais fontes de recursos vinculadas à saúde: recursos próprios vinculados à saúde pela EC-29; transferências federais voltadas para o custeio da média e alta complexidade do SUS; transferências federais voltadas para a atenção básica (PAB Fixo e PABVariável). Particularmente, o agrupamento 3, formado por 29 municípios situados predominantemente no Nordeste, é aquele que apresenta maior dependência aos recursos voltados para a atenção básica. Esses municípios, portanto, 
sofrem importante influência dos programas federais quanto aos condicionantes na utilização de recursos.

Em terceiro lugar, os dados indicam que o financiamento descentralizado do SUS remete a uma complexa relação de interdependência fiscal das três esferas de governo. Entretanto, para a maioria dos governos municipais de grande porte populacional, as principais fontes de financiamento da saúde provêem da União. O estreitamento da relação entre municípios e estados no financiamento da saúde se dá em decorrência do ICMS, e não por meio de mecanismos de transferências condicionadas a finalidades de gasto. Dessa forma, a possibilidade de interferência das Secretarias Estaduais de Saúde quanto à expansão dos recursos exclusivos da saúde é mais limitada, atrelada aos recursos repassados pela União e ainda muito pouco explorada.

Por último, se a diversidade de fontes sugere múltiplos caminhos na origem dos recursos orçamentários orientados para suprir as necessidades de gastos em saúde no âmbito local, existem muitos entraves para a redistribuição de tributos e para expansão efetiva de receitas nos municípios analisados.

As análises indicam que o PAB Variável, em 2005, propiciou significativa redistribuição de recursos na saúde privilegiando municípios situados em regiões carentes. No entanto, o volume de recursos transferido não foi suficiente para romper com as desigualdades inter e intraregionais das receitas públicas municipais, nem com a situação desfavorável dos municípios com até 200 mil habitantes. Ambas são decorrentes da menor arrecadação direta de impostos e do volume de apropriação das transferências dos estados (ICMS).

Por outro lado, a possibilidade de redistribuição do PAB Variável associa-se à capacidade de adesão do município às regras estabelecidas pelo Ministério da Saúde. No caso do PSF, se um município já tiver atingido o máximo de cobertura estabelecido, não haverá mais possibilidade de expansão de recursos por esse caminho.

Aqui se configura um dos principais dilemas das regras implantadas até 2005 para distribuição dos recursos do SUS: quanto maior for a adesão dos municípios aos programas nacionais, cuja implantação é induzida pelo gestor federal por meio de incentivos financeiros, menor será o efeito redistributivo que esses incentivos propiciam. Isso sugere a necessidade da adoção de novas estratégias de equalização fiscal mais permanentes e efetivas no âmbito da atenção básica.

Mas outra e importante limitação se coloca à redistribuição dos recursos fiscais destinados exclusivamente ao financiamento da saúde nos grandes centros urbanos. Um dilema intrínseco da política de saúde. Os recursos federais do SUS não podem deixar de seguir a oferta extremamente concentrada de serviços de saúde especializados e de alta complexidade, que se localizam justamente nos municípios com maior capacidade de apropriação das bases tributárias e, por isso, os principais arrecadadores e beneficiários dos mecanismos de devolução de tributos. Esses municípios servem, na maioria das vezes, como pólos de referência para atendimento de pacientes oriundos de outras localidades e têm crucial importância para a garantia da integralidade do cuidado à saúde.

É preciso enfatizar que, na falta de investimento público mais significativo, a expansão das receitas voltadas para a média e alta complexidade pode ser reivindicada pelos municípios por meio do credenciamento de novos serviços privados, o que estimula a privatização da oferta de maior complexidade no SUS. Além disso, embora tenham ocorrido incrementos das parcelas de média e alta complexidade transferidas aos municípios, as mudanças dos tetos financeiros não parecem acompanhar o crescimento da rede de serviços credenciada e as elevações de custos dos serviços ocorridas nas últimas décadas no país 16 .

Em síntese, mesmo com as limitações associadas à natureza do próprio fluxo - uma partilha que se destina a fins específicos, marcada por importantes condicionantes no gasto - dado a importância que têm nos orçamentos municipais, as transferências do SUS acabam proporcionando um efeito não desprezível de diminuição das diferenças nas receitas disponíveis para a saúde. Mas, se houve avanços, a pesquisa sugere que os mecanismos implantados são frágeis e temporalmente limitados, pois não alteraram as condições institucionais dos municípios com mais de 100 mil habitantes. Os entraves observados estão relacionados às questões endógenas da política de saúde (regras para a distribuição dos recursos setoriais), mas, principalmente, são frutos do sistema mais geral de repartição de competências e partilha de receitas públicas não atreladas exclusivamente ao setor da saúde. A discussão do financiamento do SUS nesses municípios remete a questões mais amplas inerentes ao federalismo fiscal brasileiro. 


\section{Resumo}

O artigo analisa as condições de financiamento do Sistema Único de Saúde (SUS) nos municípios brasileiros com mais de 100 mil habitantes, procurando aferir os resultados das regras que definem os recursos vinculados à saúde nos orçamentos municipais. Para alcance dos objetivos, foi constituída uma base de dados a partir das receitas declaradas por meio do Sistema de Informações de Orçamentos Públicos em Saúde (SIOPS) em 2005. Os valores informados foram comparados e correlacionados por localização geográfica dos municípios. Aplicou-se, ainda, a técnica de análise de agrupamentos (cluster analysis) para a conformação de grupos homogêneos segundo condições de financiamento em saúde. Verificaram-se perfis variados de receitas municipais nas diversas regiões e estados do Brasil e distintos graus de dependência orçamentária dos municípios às principais fontes de recursos vinculados. Embora a diversidade de fontes indique múltiplos caminhos para obtenção de recursos, o estudo sugere alguns entraves para o financiamento da saúde nos municípios de grande porte no país.

Financiamento em Saúde; Política de Saúde; Sistema Único de Saúde

\section{Referências}

1. Departamento de Atenção Básica, Secretaria de Políticas de Saúde, Ministério da Saúde. Indicadores de monitoramento do PSF em grandes centros urbanos. http://www.saude.gov.br/proesf (acessado em 01/Jan/2005).

2. Caetano R, Dain S. O Programa de Saúde da Família e a reestruturação da atenção básica nos grandes centros urbanos: velhos problemas, novos desafios. Physis (Rio J.) 2002; 12:11-21.

3. Escorel S, Giovanella L, Mendonça MH, Magalhães R, Senna M. Avaliação da implantação do Programa de Saúde da Família em dez centros urbanos: síntese dos principais resultados. Brasília: Ministério da Saúde; 2002.

\section{Colaboradores}

As duas autoras conceberam, estruturaram e revisaram o artigo. L. D. Lima foi responsável pela redação do trabalho e processamento dos dados. C. L. T. Andrade participou da interpretação dos dados, elaboração de tabelas e figuras além da redação do artigo.

\section{Agradecimentos}

A pesquisa foi financiada com recursos do programa Propostas de Pesquisas Avaliativas em Atenção Básica (Edital MCT/CNPq/MS-DAB/SAS no. 049/2005), apoiado pelo Departamento de Atenção Básica da Secretaria de Atenção à Saúde do Ministério da Saúde e pelo Conselho Nacional de Desenvolvimento Científico e Tecnológico, Ministério de Ciência e Tecnologia.
4. Viana ALA, Silva HP. Saúde em grandes centros. In Viana ALA, Elias PEM, Ibañez N, organizadores. Proteção social: dilemas e desafios. São Paulo: Editora Hucitec; 2005. p. 189-219.

5. Viana ALA, Rocha JSY, Elias PE, Ibañez N, Novaes MHD. Modelos de atenção básica nos grandes municípios paulistas: efetividade, eficácia, sustentabilidade e governabilidade. Ciênc Saúde Coletiva 2006; 11:577-606.

6. Pereira ATS, Campelo ACFS, Cunha FS, Noronha J, Cordeiro H, Dain S, et al. A sustentabilidade econômico-financeira do PROESF em municípios do Amapá, Maranhão, Pará e Tocantins. Ciênc Saúde Coletiva 2006; 11:607-20. 
7. Viana ALd'A. Atenção básica e dinâmica urbana nos grandes municípios paulistas, Brasil. Cad Saúde Pública 2008; 24 Suppl 1:S79-90.

8. Machado CV, Lima LD, Viana LS. Configuração da Atenção Básica e do Programa Saúde da Família em grandes municípios do Rio de Janeiro, Brasil. Cad Saúde Pública 2008; 24 Suppl 1:S42-57.

9. Lima LD. Federalismo, relações fiscais e financiamento do Sistema Único de Saúde: a distribuição de receitas vinculadas à saúde nos orçamentos municipais e estaduais. Rio de Janeiro: Museu da República; 2007.

10. Lima LD. Conexões entre o federalismo fiscal e o financiamento da política de saúde no Brasil. Ciênc Saúde Coletiva 2007; 12:511-22.

11. Mendes EV. Uma agenda para a saúde. São Paulo: Editora Hucitec; 1999.

12. Starfield B. Atenção primária: equilíbrio entre necessidades de saúde, serviços e tecnologia. Brasília: Organização das Nações Unidas para a Educação, a Ciência e a Cultura/Ministério da Saúde; 2002.
13. Hamilton LC. Regression with graphics: a second course in applied statistics. Belmont: Duxbury; 1992.

14. Johnson RA, Wichem DW. Applied multivariate statistical analysis. New Jersey: Prentice-Hall; 1988.

15. Prado S. Distribuição intergovernamental de recursos na federação brasileira. In: Rezende F, Oliveira FA, organizadores. Descentralização e federalismo fiscal no Brasil: desafios da reforma tributária. Rio de Janeiro: Konrad Adenauer; 2003. p. 41-125.

16. Machado CV. Direito universal, política nacional: o papel do Ministério da Saúde na política de saúde brasileira de 1990 a 2002. Rio de Janeiro: Museu da República; 2007.

Recebido em 13/Fev/2009

Aprovado em 23/Jun/2009 